\title{
HACIA UN NUEVO PLURALISMO EN LA TELEVISIÓN EUROPEA
}

\author{
Ma Teresa la Porte Y Teresa Sádaba \\ Profesoras en la facultad de Comunicación. Universidad De Navarra
}

\section{Resumen}

El objetivo de este estudio es proponer un nuevo concepto de pluralismo que garantice su presencia efectiva en los medios de comunicación. De acuerdo con este concepto, se sugieren tres indicadores para medir el grado de pluralismo: la presencia de los diferentes grupos sociales o políticos, la naturaleza de su intervención y las características de la imagen difundida.

La muestra seleccionada procede de cinco países europeos: Alemania, Francia, Italia, España y Reino Unido. La metodología utilizada es el análisis de contenido de los informativos. El estudio demuestra la insuficiencia del concepto y medición actual presente en las legislaciones europeas.

\section{Palabras clave}

Pluralismo, Televisión Pública, Informativos, Europa, legislación nacional.

\section{Abstract}

The aim of this study is to propose a new concept of pluralism to guarantee its real presence in the media. Taking in account this concept, three main indicators are used: the presence of the different social and political groups, the nature of their intervention and the characteristics of the image transmitted.

The selected sample comes from five European countries: Germany, France, Italy, Spain and the United Kingdom. Pluralism in the news is evaluated through the analysis of quantitative content. The study shows the concept and measure of pluralism in the European national laws is unsatisfactory.

\section{Key words}

Pluralism, Public Television, Europe, News Content, national legislation. 


\section{INTRODUCCIÓN}

La defensa del pluralismo vuelve a ser un tema controvertido en el entorno europeo. En ocasiones anteriores, el debate se generó como consecuencia de los riesgos que provocaba la concentración de medios de comunicación. La novedad de la polémica actual es que retoma la discusión sobre las funciones de la televisión pública, garante tradicional del pluralismo, cuestionando su comportamiento en un mercado audiovisual dominado por criterios comerciales.

Así, por ejemplo, en España, el nuevo gobierno incluye en su programa político la creación de un Consejo que regule el contenido audiovisual, siguiendo en parte el ejemplo catalán. En Alemania se revisa el sistema establecido para garantizar el pluralismo en el medio público por su insuficiencia para atender las diferencias territoriales. Francia cuestiona la excesiva rigidez de la medición del pluralismo en los contenidos de la televisión pública mientras que en el Reino Unido, el caso Kelly ha provocado una fuerte discusión en torno a la independencia del servicio público. Por último, también Italia acaba de plantearse la efectividad del concepto de servicio público contemplado en la legislación.

La causa del debate, con algunos matices, es prácticamente la misma en todos los casos: la televisión pública ha adoptado algunas prácticas propias de la televisión comercial como medida necesaria para subsistir en un mercado cada vez más competitivo. Este contagio provoca, en último término, que su oferta sea muy similar a la de la televisión privada. La pregunta sobre la necesidad de su existencia está latente tanto entre los contribuidores como entre sus directos competidores.

Sin embargo, las instancias políticas europeas coinciden mayoritariamente en la defensa del servicio público. La revisión actual se limita a plantear la necesidad de redefinir su función, diseñar nuevas vías de financiación y perfeccionar los instrumentos legales que garanticen la defensa de los valores que el medio público tiene asignados.

El debate se origina, no obstante, sin excesivo fundamento empírico. Sólo existen evidencias que justifiquen esta revisión en los métodos de financiación, donde las prácticas de la televisión pública son fácilmente verificables. La denuncia sobre la reducción del pluralismo en los contenidos es, en la mayor parte de los casos, puramente intuitiva, siendo muy insuficientes también los métodos de medición de la presencia de esos valores en la programación.

El presente trabajo pretende aportar datos cuantitativos sobre las diferencias entre el pluralismo de la televisión pública y el de la televisión comercial para evaluar el comportamiento del servicio público en el ámbito europeo y su aportación real en defensa de estos principios. A estos efectos, la muestra seleccionada son los cinco países miembros con audiencias mayoritarias: Alemania, España, Francia, Italia y Reino Unido.

El estudio propone también un sistema de medición del pluralismo basado en las fuentes utilizadas en los informativos del prime time que permite valorar la variedad de los enfoques, la representación cualitativa de los diversos sectores de la política y de la sociedad y el grado de acceso al público. 


\section{LEGISLACIÓN EN TORNO AL PLURALISMO}

Desde el punto de vista teórico, son numerosas las investigaciones que tratan el fenómeno de la televisión pública en un mercado competitivo y globalizado. En este contexto, la función de la televisión pública requiere una redefinición que afecta, en primer lugar, a las legislaciones sobre medios de los países, donde actualmente ya se está afrontando el debate.

Existe un acuerdo, desde el punto de vista académico y normativo, sobre la necesidad de que los canales públicos asuman la defensa del pluralismo, fundamento de cualquier sistema democrático. Donde reside la complejidad es en las propias definiciones de pluralismo y de la manera adecuada de medirlo.

Respecto al marco jurídico del pluralismo, las legislaciones democráticas contemplan en sus distintos niveles normativos el pluralismo informativo como cauce de expresión de las diversas manifestaciones de la sociedad. Algunos países elevan el pluralismo al rango constitucional, otros lo hacen en la normativa interna de los medios, pero en cualquiera de los casos, el pluralismo informativo se asocia a dos conceptos fundamentales: representatividad y oferta plural.

Por oferta plural se entiende que los medios tienen que plantear una oferta variada, no monopolística, como requerimiento básico del mercado de ideas plural. La multiplicidad de canales, operadores, productores, etc., parece ser la garantía de este pluralismo en el panorama europeo.

El pluralismo como representatividad supone atender a las distintas sensibilidades sociales permitiendo su acceso a los contenidos de los medios. Desde la teoría política, se ha entendido la representación como la copia fidedigna del cuerpo representado en el representante, es decir, por ejemplo que la composición del legislativo corresponda con exactitud a la de toda la nación. Para ello se recurre a muestreos y sistemas proporcionales en un intento de hacer la copia del original, recogiendo todas sus características (Pitkin, 1985)'. En el caso de los medios, habría que dar cabida por tanto a todas las voces sociales que integran la comunidad en la que se opera. Debido al origen de las televisiones europeas, este concepto suele reflejarse en la necesidad de que todas las voces del espectro político tengan representación en la programación de las cadenas ${ }^{2}$.

\footnotetext{
En la práctica se han intentado distintas fórmulas, que sobre todo inciden en características de territorio y población. Según la magnitud de los distritos electorales, sus límites territoriales y el prorrateo o relación entre el número de electores por escaño la fidelidad de la representación entendida como fotografía del electorado puede variar. También dependiendo del sistema proporcional o distributivo utilizado (mayores restos, mayores medias, etc.). Para profundizar sobre este tema, véase: Vallés y Bosch, 1997.

2 Debido a que el pluralismo en las legislaciones ha adoptado una visión bastante política, para hablar de la representación de otro tipo de grupos sociales parece que impera ahora la noción de diversidad cultural. Este es el concepto que se incorpora en la doctrina comunitaria respecto a los medios y su impacto social (La Porte y Sádaba, 2002). En esta misma línea, también desde la UNESCO se ha instado a la defensa del pluralismo en los medios. (Cfr. Informe Mundial sobre la cultura 2000/2001. Diversidad Cultural, Conflicto y Pluralismo. Ediciones UNESCO, 2001
} 
Así sucede en el caso de España. La Constitución de 1978 consagró el principio del pluralismo informativo en su artículo 20: "La ley regulará la organización y el control parlamentario de los medios de comunicación social dependientes del Estado o de cualquier ente público y garantizará el acceso a dichos medios de los grupos sociales y políticos significativos, respetando el pluralismo de la sociedad y de las diversas lenguas de España".

Según el Estatuto de la Radio y Televisión Española, Ley 4/1980, "se respetará como principio básico el pluralismo político, religioso, social, cultural y lingüístico". Su artículo diez insta a "Determinar semestralmente el porcentaje de horas de programación destinadas a los grupos políticos y sociales significativos, fijando los criterios de distribución entre ellos en cumplimiento de lo establecido en el artículo 20 de la Constitución".

No obstante, sólo el ámbito político ha tenido una legislación que, efectivamente, desarrolle las fórmulas adecuadas para garantizar el pluralismo. Dicha ley, la Ley Orgánica 5/1985, de 19 de junio, de Régimen Electoral General (LOREG), modificada en dos ocasiones por la Ley Orgánica 13/1994, de 30 de marzo y por Ley Orgánica 8/1999 de 21 de abril, es la que regula la actuación de los medios públicos en tiempo de campaña electoral (principalmente, respecto al reparto de espacios gratuitos) y sólo tiene por lo tanto una incidencia política ${ }^{3}$.

La aparición de las cadenas privadas en 1988 no supone una reforma normativa y pese a que su adjudicación se hace "atendiendo a los criterios del pluralismo" (en cuanto oferta plural) nada se regula sobre sus contenidos.

En Alemania, la Ley Fundamental, entre sus artículos no susceptibles de revisión, asegura la libertad de expresión como un derecho de todos los ciudadanos, y garantiza también la accesibilidad de las fuentes. El Estado, de acuerdo con la constitución, mantiene una neutralidad respecto a los contenidos televisivos.

El Estado delega la responsabilidad de la radioteledifusión a los Estados Federados (Decisión de la Corte Constitucional de 1961). Son los Estados Federados quienes autorizan, organizan la financiación y establecen normas sobre el contenido de las cadenas. La federación se reserva las competencias de telecomunicaciones y su reglamentación técnica, así como las cuestiones relacionadas con la propiedad intelectual.

Para garantizar el pluralismo territorial y cultural, entre las distintas cadenas de los Estados, existe un acuerdo para subvencionar a los más pequeños para que puedan tener sus propias emisoras frente a los grandes (aunque esto actualmente, y debido a cuestiones económicas, está muy cuestionado).

\footnotetext{
3 La LOREG se basa en dos principios fundamentales: no discriminación y acceso a medios públicos. Durante la campaña electoral los partidos, federaciones, coaliciones y agrupaciones que concurran a las elecciones tienen derecho a espacios gratuitos de propaganda en las emisoras de televisión y de radio de titularidad pública. Estos espacios se distribuyen proporcionalmente al número de votos obtenidos en las anteriores elecciones equivalentes.
} 
En el caso de las cadenas públicas existe un Consejo de Radioteledifusión que vela por el pluralismo. Por eso, está compuesto por representantes de los partidos, iglesias, sindicatos, organizaciones de mujeres, juventud...

En Alemania existe el principio de complementariedad. La condición para que haya televisiones privadas es que el servicio público de televisión provea del servicio básico universal. A su vez, la televisión privada debe facilitar el pluralismo.

La ley $n^{\circ} 86-1067$ de 30 septiembre de 1986 relativa a la libertad de comunicación (modificada el 1 agosto de 2000) aboga por el pluralismo para establecer canales televisivos en Fran$\mathrm{cia}^{4}$. En este sentido, se otorga un papel principal al Conseil Supérieur de l'Audiovisuel (CSA) para garantizar el pluralismo informativo. Su ámbito de actuación no se limita a las emisoras privadas, sino que atiende también a las públicas. El artículo primero de dicha ley habla de que la libertad de comunicación sólo puede estar limitada, entre otras cosas, por "el carácter pluralista de la expresión de las corrientes de pensamiento". En su artículo 13 anuncia que esto principalmente sucederá en el ámbito político, del que el CSA da cuenta mensualmente sobre su aparición.

Por el artículo 55 de dicha ley, se entiende que el CSA puede definir las modalidades de intervención de formaciones políticas, sindicatos, organizaciones profesionales, etc. También se garantizan emisiones de carácter religioso de los principales cultos que se practican en Francia.

El CSA contaba, hasta el año 2000, con la denominada regla de los "Tres Tercios" para controlar el pluralismo. Esta regla consistía en otorgar una presencia repartida del siguiente modo: 1/3 para el gobierno, 1/3 para la mayoría parlamentaria, 1/3 para la oposición. Durante el año 2000, la revisión de la normativa llevó a nuevos modos de evaluación del pluralismo, puesto que todos los actores implicados coincidían en que la regla de los Tres Tercios era excesivamente reduccionista y rígida.

El nuevo principio establecido hace que los editores respeten el equilibrio entre el tiempo de intervención del gobierno, de la mayoría parlamentaria y de la oposición, asegurando condiciones similares de programación. Además, también tienen que vigilar por tiempos de intervención de partidos que no tienen presencia en el Parlamento.

En periodo electoral, el Consejo tiene un papel muy relevante y se puede afirmar que efectivo, ya que su mera presencia y poder coercitivo hace que se respeten de manera estricta los tiempos establecidos. La misma ley especifica como requerimiento para establecer canales televisivos el respeto por el pluralismo.

${ }^{4}$ El artículo 40 de la ley del 30 de septiembre de 1986 recientemente modificado, que prohíbe a una cadena hertziana ser propiedad de más del $20 \%$ de personas externas a la Unión Europea. 
Actualmente, en Italia se está cursando una nueva ley de reforma del sistema radiotelevisivo, denominada ley Gradassi (nombre del ministro que la encabeza). Teniendo en cuenta que la garantía de la libertad y del pluralismo son los principios fundamentales del sistema radiotelevisivo italiano, tal y como afirma su legislación, en el año2000, mediante ley se expuso que todas las fuerzas políticas tienen igualdad de oportunidades para el acceso a la televisión. En los programas informativos se tendrá acceso, teniendo en cuenta la representación política, en el resto de contenidos políticos (tertulias, debates, información preelectoral) no hay jerarquía de tiempos.

En el caso de los informativos, se distingue entre: tiempo de la noticia (el que otorga el periodista para ilustrar las informaciones), tiempo de la palabra (habla directamente con su voz), tiempo de antena (suma entre el tiempo de la noticia y el tiempo de la palabra).

Para las cadenas públicas, existe un órgano de control parlamentario (una Comisión de vigilancia bicameral) y en las privadas existe una Autoridad de Garantía de Comunicaciones.

El modelo británico responde a un tipo de pluralismo externo. En Reino Unido, elservicio público de televisión es prestado básicamente por la BBC (British Broadcasting Corporation) corporación de derecho público que explota dos programas de televisión por ondas analógicas y 7 programas de TDT. Su habilitación para emitir procede de un Real Decreto, válido por diez años, y que fue renovado en $1996^{5}$.

En el White Paper británico, "The Future of the BBC. Service the nation competing world-wide" (1994) se recogen diez objetivos que el gobierno considera que debe cumplir la BBC (art. 2.6), entre ellos: promover la diversidad; reflejar la identidad nacional; y mantener la independencia informativa; facilitar el acceso a todos los sectores de la sociedad. En su capítulo 4 se expone que la "diversidad y el pluralismo se pueden conseguir mediante las propuestas de otros capítulos que aseguran un alto dinamismo de mercado y la máxima calidad de contenidos. Para complementar estas propuestas, este capítulo reafirma la preocupación del gobierno por la producción independiente, la diversidad de la provisión de las noticias de televisión y los servicios donde concurren intereses regionales, locales y culturales".

En un intento de aproximación a los dos niveles de pluralismo, en 2003 la Communications Act distingue el pluralismo, que lo identifica con la posibilidad de elección de los diferentes ofertantes, de la diversidad, que se aplica a los distintos tipos de programas.

\footnotetext{
5 Las obligaciones específicas de servicio público impuesta sala BBC se encuentran en un Agreement en el que el Gobierno establece las condiciones básicas para el desarrollo de su actividad. La financiación de la prestación de este servicio público se lleva a cabo mediante el cobro de un canon (los programas de servicio público de la BBC analógicos emitidos en Reino Unido no admiten publicidad). El control de la actividad de la BBC le corresponde a un organismo interno, el Boardof Governors,compuesto de doce miembros designados por el Gobierno. Para las televisiones comerciales en 1956 se establece la Independent Television Commission. Por otra parte, la Broadcasting Standards Commission se encargaba de velar por el cumplimiento de las normas relativas a la decencia y el buen gusto en los contenidos, tanto de la televisión pública como de la privada. Desde 2004, un solo organismo (OFCOM) reúne a todas las comisiones competentes sobre los medios de comunicación.
} 
Completan el panorama del mercado televisivo distintas cadenas que pretenden garantizar el pluralismo. La ITV (Independent Television), Channel 3, cubre los intereses regionales; Channel 4 se dirige a las minorías, satisface necesidades culturales y da juego a productores independientes, y Channel 5, la última televisión aprobada, tiene los rasgos típicos de un canal comercial (comenzó de 30 marzo de 1997).

En definitiva, las legislaciones sobre el pluralismo tienden a abordar los dos modos de regular este tema desde el punto de vista informativo, en cuanto oferta plural y en cuanto a representación. Estos dos conceptos son los que han vertebrado los modos de entender el pluralismo y que a continuación se resumen.

\section{MARCO TEÓRICO DEL ESTUDIO}

El trabajo de investigación adopta el concepto de pluralismo utilizado por el Consejo de Europa. Esta definición incluye todas las dimensiones del pluralismo que afectan a los medios de comunicación. También es especialmente oportuna porque esta institución actúa en el mismo ámbito geográfico seleccionado en este estudio.

"Desde el punto de vista de las concentraciones de los medios, la noción de pluralismo debe ser entendida como la posibilidad de que una larga gama de valores, opiniones, informaciones e intereses de orden social, político y cultural puedan encontrar el medio de manifestarse a través de los medios de comunicación de masas. El pluralismo puede ser interno, a través de una amplia gama de valores, opiniones, informaciones e intereses que encuentran un vehículo de expresión en el seno de un organismo determinado del sector de los medios, o bien externo a través de un cierto número de estos organismos, cada uno de ellos expresando un punto de vista particular" (Consejo de Europa, 1994:8).

De este modo, pluralismo interno y pluralismo externo son los dos modos de concebir el pluralismo que coinciden de modo aproximado con lo que en las legislaciones se concebía como representación y oferta plural respectivamente.

El pluralismo externo está relacionado con la concentración del mercado, mientras que el interno se vincula a la diversidad de ideas y opiniones en los contenidos. Con cierta frecuencia, se ha entendido que el primero afecta al segundo, en cuanto que un mercado más concentrado puede limitar el pluralismo de los contenidos. Esta hipótesis se ha contrastado con recientes estudios que señalan que la concentración afecta a los niveles financieros y productivos, y no siempre a los contenidos (Sánchez-Tabernero y Carvajal, 2002).

En torno a este debate aparecen tres escuelas de investigación diferenciadas. En primer lugar, la escuela crítica aboga por un proteccionismo que evite la excesiva concentración, argumen- 
tando que los productos mediáticos son productos culturales (Wright, 1994; Shaughnessy, 1990; Burgelman y Pauwels, 1992; Machet, 1998). En el lado opuesto, la escuela liberal, apuesta por el libre mercado de ideas que lleva a su propia regularización y equilibrio. En tercer lugar, la escuela moderada, introduce nuevas variables al debate, explicando que en la defensa del pluralismo, no sólo se puede tener en cuenta los factores del mercado, sino que hay que atender a otros elementos como las propias funciones de los medios, el concepto que tienen de sí mismos, su línea editorial, etc. (Toussanit, 1996; Picard, 2002; Albarran, 2002; Nieto e lglesias, 2000; Doyle, 2002). Por lo tanto, el punto central del debate se traslada de la titularidad de los medios a sus contenidos, enfoque que también adopta este trabajo.

Teniendo en cuenta las aportaciones realizadas, la hipótesis de partida del presente estudio se sitúa en el denominado pluralismo interno y atiende a los argumentos de la escuela moderada en cuanto que su enfoque principal son los contenidos.

En este contexto la investigación propone sin embargo un nuevo concepto de pluralismo interno que contemple no sólo la visibilidad de los diversos actores políticos y sociales, sino su tratamiento en los medios de comunicación. Este nuevo concepto refleja de manera más acertada la aportación específica de los medios al pluralismo, que no se limita a garantizar la presencia o representación sino que también añade un enfoque determinado a dicha representación.

Así mismo, se parte de la idea de que la televisión pública tiene una específica misión de salvaguarda del pluralismo y actúa, por lo tanto, como factor de equilibrio ante los riesgos de la excesiva comercialización.

De este modo, se supone que los contenidos de la televisión pública tienen elementos diferenciadores respecto a la privada.

\section{PROPUESTA DE UN MODELO DE EVALUACIÓN DEL PLURALISMO}

De acuerdo con el concepto de pluralismo asumido, el modelo que se propone para la valoración de su presencia en los informativos está sustentado sobre tres indicadores: representación de los grupos sociales o políticos, naturaleza de su intervención y características de la imagen difundida. Estos tres indicadores se recogen en el código desglosado en las variables que se describen a continuación.

La representación de los grupos sociales o políticos se valora por la frecuencia de su aparición. La presencia en el medio implica visibilidad en la esfera pública y también, por tanto, acceso a la audiencia: tanto la visibilidad pública como la posibilidad de contactar con el resto de los ciudadanos son rasgos que garantizan la existencia de una sociedad plural real y efectiva. 
Sin embargo, no parece que la mera presencia sea suficiente para valorar el grado de pluralismo. Es necesario considerar también la naturaleza de la intervención, esto es, si el representante tiene voz propia o habla en su lugar el periodista $u$ otra fuente. Se entiende que en el segundo caso hay una disminución del protagonismo, con el consiguiente deterioro de la autoridad como actor político o social.

Junto al tipo de intervención, es interesante valorar también la imagen positiva o negativa que resulta del tratamiento periodístico que se da al protagonista evaluado: una presencia excesiva con una imagen negativa perjudicaría la posición política o grupo cultural representado, mientras que una presencia breve tratada de manera positiva resultaría mucho más eficaz en términos de incidencia pública.

El principal elemento de análisis son los protagonistas de las noticias, las figuras políticas o sociales que intervienen en la información. Ese protagonismo se entiende en un doble sentido: o bien se refiere al actor principal en torno al cual se desarrolla la información, o bien es la fuente principal de quien se solicita declaraciones.

Sin embargo, el aspecto que interesa del protagonista no es su valor personal sino su función como representante de una institución o grupo social. En una sociedad democrática son las instituciones o grupos sociales, no los individuos, los que aglutinan las diversas opiniones y los que reflejan la diversidad cultural.

En relación con los criterios para la selección de la muestra, los datos se han obtenido de los tres sectores más significativos para la evaluación del pluralismo: el sector político, el sector social y el sector cultural.

En el sector político, el código distingue los representantes nacionales de los internacionales y permite cuantificar la aparición de cada uno de ellos. Se entiende que el pluralismo será mayor en la medida en que exista un equilibrio proporcionado entre la presencia de estos dos tipos de representantes. Este resultado permite además deducir el grado de localismo o visión nacional de la información.

Los representantes internacionales se dividen a su vez reflejando los centros de decisión con mayor incidencia en la política exterior europea: esto es, Estados Unidos y la Unión Europea. La frecuencia con que sus portavoces o representantes aparecen como fuentes informativas refleja la dependencia de uno u otro centro de poder.

El análisis de representantes internacionales diferencia también las instituciones que detentan el protagonismo en las relaciones políticas, evaluando la presencia de la institución militar, la del gobierno y la de los ciudadanos. En las relaciones con los Estados Unidos, centra- 
das en ese momento en el conflicto de Irak, cada uno de esos grupos tiene un enfoque diferente del conflicto: una presencia equilibrada de todos ellos garantizaría el pluralismo mientras que un desequilibrio a favor de alguno de los sectores lo pondría en riesgo.

Un criterio similar se ha aplicado a la hora de valorar las tensiones internas en el seno de la Unión Europea, en este caso simbolizadas por los gobiernos de los estados miembros, por las instituciones de la Unión y por los propios ciudadanos europeos. La intervención mayoritaria de alguno de estos sectores revelaría también una posición sesgada sobre la dinámica de poder en la política europea.

En relación con los representantes nacionales, interesa la variedad de la aparición de los líderes políticos, clasificados según tres opciones: partido gobernante, partido de la oposición, tercera fuerza política. Junto a la cuantificación de la presencia, en la mayor parte de los países comunitarios exigida por ley, también se estudia a este nivel la valoración positiva, negativa o neutra de su tratamiento informativo.

En relación con el sector cultural y social, la metodología adoptada tiene las siguientes características. Las minorías culturales y sociales responden a una definición amplia que pueda dar cabida a todos los grupos significativos que conformen las diferentes sociedades estudiadas en el proyecto. Como de los protagonistas anteriores, de cada uno de ellos interesa conocer la naturaleza de su intervención, si lo hacen o no con voz propia, y, de nuevo, la imagen informativa que se deriva de la cobertura.

\section{MEDICIÓN DEL PLURALISMO EN LOS INFORMATIVOS EUROPEOS}

El análisis de los informativos de prime time en Alemania, Francia, Italia, España y Reino Unido, produce un total de 1346 noticias, con un reparto total entre cadenas públicas y privadas bastante equitativo en todos los países analizados.

Reparto en cadenas por países (en \%)

\begin{tabular}{|l|l|l|l|l|l|}
\hline & Alemania & Francia & lialia & España & R.Unido \\
\hline Público & $50,5 \%$ & $51,7 \%$ & $58,3 \%$ & $51,1 \%$ & $58,2 \%$ \\
\hline Privado & $49,5 \%$ & $4,3 \%$ & $41,7 \%$ & $48,9 \%$ & 41,8 \\
\hline
\end{tabular}

Las cadenas escogidas de cada uno de los sectores responden a un criterio de audiencias globales de los canales (y no específicamente de los informativos). Es decir, se ha escogido el canal público y privado con mayor audiencia entre los años 2000 y 2002. 
Cadenas analizadas por países

\begin{tabular}{|lllll|}
\hline $\begin{array}{c}\text { PAIS } \\
\text { (FUENTE) }\end{array}$ & CANAL & & $\begin{array}{l}\text { \%Audiencia } \\
\text { 2001-2002 }\end{array}$ & $\begin{array}{l}\text { \%Audiencia } \\
2000-2001\end{array}$ \\
\hline FRANCIA & PUB & TF1 & $32.5(2002)$ & $33(2001)$ \\
Mediametrie & PRIV & France 2 & 20.8 & 21 \\
\hline ALEMANIA & PUB & ARD & $13.4(2001)$ & $13.4(2001)$ \\
AGF/Gfk & PRIV & RTL & 14.9 & 14.9 \\
\hline ITALIA & PUB & RAI 1 & $25.7(2001)$ & $25.7(2001)$ \\
Auditel & PRIV & Canale 5 & 23.9 & 23.9 \\
\hline ESPAÑA & PUB & TVE 1 & $24.9(2002)$ & $24.5(2000)$ \\
RTVE/Sofres & PRIV & T5(A3) & 20.1 & $22.3 / 21.5$ \\
\hline R. UNIDO & PUB & BBC 1 & $26.4(2002)$ & $26.6(2001)$ \\
ITC & PRIV & ITV & 23 & 29.8 \\
\hline
\end{tabular}

Fuente: The Media Map Yearbook. 2003, 2002. CIT.

El periodo de muestra (seleccionado aleatoriamente) fue la semana del 22 al 28 de septiembre de 2003. Durante aquellos días, los acontecimientos más destacados fueron los siguientes: estalla un coche bomba en la sede de la ONU en Bagdag, continúan las detenciones por el asesinato de la ministra sueca Anna Lindh (ocurrido el 10 de septiembre), envío de refuerzos a Irak por parte de Estados Unidos mientras continúa la búsqueda de las armas de destrucción masiva, continúan las investigaciones sobre el caso Kelly en Londres, Blair anuncia un posible referéndum sobre el euro en su país, Italia sufre el mayor apagón de su historia, se detiene a Alexander King (supuesto asesino de Rocío Wanninkhof y Sonia Carabantes), en Alemania tienen lugar comicios regionales.

En el análisis de los indicadores establecidos del pluralismo (representación de los grupos sociales y políticos, naturaleza de las intervenciones y tratamiento) se han distinguido tres niveles.

- En primer lugar se analiza el pluralismo de los distintos grupos representados en cada uno de los países, comparando en todos ellos el comportamiento de la cadena pública frente al de la privada. De este modo, se quiere comprobar si existen tendencias nacionales.

- En segundo lugar, se examinan los datos globales de la muestra, sin diferenciar los países y distinguiendo únicamente por propiedad del medio, para demostrar si hay una uniformidad de planteamientos en el sector público y en el privado en el conjunto de la UE.

- Finalmente, el análisis aborda el examen global de los datos tomados sin establecer ninguna variable independiente y como muestra única, de manera que se pueda llegar a concluir algunos parámetros de los grupos representados que más presencia tienen de manera global en los países escogidos. 


\section{ANÁLISIS COMPARATIVO ENTRE LOS PAÍSES EUROPEOS}

La muestra de las informaciones de España revela pocas diferencias en la distribución de noticias por sector. De las 372, un 51,1\% pertenecen a Televisión Española y un 48,9\% a Tele Cinco. Las noticias tienen principalmente un marcado localismo, puesto que proceden del propio país en un $71,2 \%$ de los casos, tanto en la cadena pública como en la privada.

Esta tendencia se confirma de nuevo al observar quiénes son los protagonistas de las informaciones, hay una clara mayor presencia de los actores nacionales respecto a los internacionales, de modo un poco más acusado en el canal público.

\section{Fuentes en España}

\begin{tabular}{|l|c|c|}
\hline & Nacional & Internacional \\
\hline Público & $18,9 \%$ & $3,7 \%$ \\
\hline Privado & $18,1 \%$ & $9,9 \%$ \\
\hline
\end{tabular}

De este modo, y dentro de los escasos momentos en los que los protagonistas internacionales tienen voz en el informativo, cabe destacar que es el gobierno de Estados Unidos quien aparece en más ocasiones que los miembros de las instituciones comunitarias (si bien los porcentajes son siempre poco significativos: $3 \%$ de fuentes americanas, $1.6 \%$ de gobiernos nacionales de la UE y un $0,9 \%$ de las instituciones europeas).

En cuanto a las apariciones o mera presencia de los protagonistas, se ratifica la misma tendencia, aunque hay que mencionar que se introducen nuevos actores a los que no se les da voz como por ejemplo sucede con el ejército americano.

Un mayor protagonismo como actores y fuentes lo tienen los ciudadanos europeos, pero como se decía anteriormente, la importancia recae sobre los actores nacionales. En este caso, es el gobierno del propio país, ya sea presentando políticas o defendiendo una postura gubernamental, el que tiene una presencia superior ligeramente más alta en la cadena pública (un $9 \%$ frente a un $7.1 \%)$, no obstante hay que señalar que este mismo reparto se produce cuando las cadenas dan voz al principal partido de la oposición.

Un examen más detenido de los enfoques de estos protagonistas nacionales es el que arroja mayores diferencias en el tratamiento de TVE y Tele 5. Cuando la visión del gobierno de la cadena pública es principalmente positiva o neutra, para la privada sucede lo contrario.

Enfoque de las fuentes nacionales en España

\begin{tabular}{|l|l|l|l|}
\hline & Positivo & Negativo & Neutro \\
\hline Público & $8,4 \%$ & $3,7 \%$ & $6,8 \%$ \\
\hline Privado & $2,2 \%$ & $9,3 \%$ & $5,5 \%$ \\
\hline
\end{tabular}


Llama la atención la total ausencia de minorías como fuente de las informaciones en los noticieros españoles.

Alemania presenta un total de 186 noticias, con un reparto casi igual entre pública y privada $(50,5 \%$ y $49,5 \%$ respectivamente). A pesar de que de nuevo, cobra mayor importancia la noticia local, en un $59,1 \%$ de los casos proceden del propio país (el porcentaje es bastante superior en la cadena pública) tienen un lugar relevante las noticias procedentes tanto de la UE, como de EEUU o el resto del mundo (esto en porcentajes muy similares).

Entre las fuentes internacionales que se mencionan, se distinguen claramente los gobiernos de los países que conforman la Unión Europea, después el gobierno de Estados Unidos y finalmente las instituciones comunitarias. De nuevo, esta tendencia resulta más acusada en el caso de la cadena pública.

Fuentes internacionales en Alemania

\begin{tabular}{|l|l|l|l|}
\hline & Estados Unidos & Países europeos & UE \\
\hline Público & $3,3 \%$ & $7,5 \%$ & $3,3 \%$ \\
\hline Privado & $6,6 \%$ & $1,1 \%$ & $1,1 \%$ \\
\hline
\end{tabular}

Respecto al gobierno de Alemania, su presencia es también superior a la de la oposición, dato que se refuerza significativamente en la cadena pública. La oposición apenas tiene presencia y los enfoques de estos protagonistas son más bien neutros.

Entre las fuentes que hablan de minorías sociales y culturales los periodistas mismos se configuran como los principales vehículos de sus demandas, y aunque dichas minorías tienen un espacio en el informativo no aparecen como fuentes de las noticias que protagonizan.

El mayor número de noticias analizadas lo presenta Francia, con 383 y un reparto también muy equitativo entre sus canales $(51,7 \%$ el público frente al $48,3 \%$ del privado). La procedencia de las noticias tiene también carácter local, pero destaca el hecho de que la procedencia de noticias de otros países europeos duplica a las que llegan de Estados Unidos. De hecho, este es el país que menos voz da a los políticos, ejército y ciudadanos americanos (apenas llega al $1 \%$ de sus fuentes).

Entre las fuentes internacionales se subraya la presencia de ciudadanos europeos como en ningún otro de los casos analizados. Los ciudadanos de la UE son fuente en casi la mitad de las noticias, de modo más llamativo en la cadena pública y con una imagen bastante positiva.

El gobierno francés ocupa un lugar preferente en las informaciones respecto al resto de fuentes nacionales. En este caso, los dos canales otorgan la misma voz a sus mandatarios, aunque en el canal privado se omite con más frecuencia a la oposición. Sin embargo, la diferencia más sus- 
tancial se encuentra en el enfoque de las noticias, puesto que en el trato al gobierno, la cadena pública inclina la balanza claramente con una visión positiva mientras que la privada parece más neutra.

Enfoques de la Fuente gubernamental en Francia

\begin{tabular}{|l|l|l|l|}
\hline & Positivo & Negahivo & Neutro \\
\hline Público & $5,6 \%$ & $3,0 \%$ & $3,0 \%$ \\
\hline Privado & $2,2 \%$ & $3,2 \%$ & $3,2 \%$ \\
\hline
\end{tabular}

Las noticias de la BBC e ITV suman un total de 153 noticias $158,2 \%$ y $41,8 \%$ respectivamente) analizadas en Reino Unido. También destaca el propio país como foco de las informaciones, seguido de la categoría "otros" donde se incluyen países de todo el mundo excepto los Estados Unidos y los pertenecientes a la Unión Europea.

Esta misma idea se ratifica en el uso de las fuentes, donde tienen una presencia abrumadora las nacionales respecto a las internacionales (que no llegan al $1 \%$ ). Nuevamente es el gobierno la fuente principal, aunque curiosamente lo es más en la cadena privada que en la pública. Sin embargo, el enfoque de estas noticias de la emisora privada con fuentes gubernamentales tiene en la mayoría de los casos un sesgo negativo.

Además del partido de la oposición aparecen los ciudadanos europeos, y al tratar sobre minorías sociales se menciona a periodistas y expertos como fuentes.

Por último, en Italia se recogieron 252 noticias, con el reparto más desigual de los vistos hasta el momento entre públicas y privadas (58,3\% la RAl y $41,7 \%$ Tele 5$)$. De la misma forma que en los casos anteriores el carácter local de la emisión de las noticias queda demostrado con un $79 \%$ de ellas emitidas desde el propio país. Destaca, en cualquier caso, el $20 \%$ de las producidas en Estados Unidos (en segundo lugar en ambas cadenas).

La presencia de fuentes internacionales sigue siendo bastante baja, y son las posturas del gobierno las que más aparecen en los informativos. Aquí, sin embargo, hay una diferencia importante entre las cadenas, puesto que la pública concede casi el doble de protagonismo al gobierno que la privada. Además, si se profundiza en el enfoque de estas noticias, cabe señalar que la RAl otorga un tratamiento positivo o neutro, mientras que el canal cinco presenta una postura neutra o negativa principalmente.

Destacan los ciudadanos como fuentes generando su opinión en un 9,5\% de los casos, el mayor de todos los analizados. También, es la única ocasión en la que la minoría social se presenta como fuente para las informaciones, aunque sea en un $1,6 \%$ en la RAI. 


\section{ANÁLISIS COMPARATIVO ENTRE LAS CADENAS PÚBLICAS Y PRIVADAS}

Si se contabilizan los datos de manera global, sin diferenciar por países, y se distingue por la propiedad del medio, se pueden observar las siguientes correlaciones.

Respecto al lugar de emisión, no hay grandes diferencias en el comportamiento de unas y otras en los cinco países. Las cadenas transmiten sus noticias desde los mismos lugares, independientemente de la propiedad.

Emisión de la noticia

\begin{tabular}{|l|l|l|l|l|}
\hline & Propio país & UE & EEUU & Otros \\
\hline Público & $70,90 \%$ & $10,40 \%$ & $7,50 \%$ & $11 \%$ \\
\hline Privado & $69,10 \%$ & $10,40 \%$ & $8,30 \%$ & $12,30 \%$ \\
\hline
\end{tabular}

Por temas tratados, sucede algo similar en todos ellos, excepto los "desastres" y las "noticias de interés humano" que tienen mayor cabida en las emisoras privadas, el resto de las cuestiones recibe una presencia informativa bastante parecida. Esta tendencia se refleja también en los protagonistas y fuentes mencionadas. La escasa presencia de actores internacionales, ya sean estadounidenses o europeos, es una pauta común. Existen ligeras diferencias en la aparición de los ciudadanos europeos, a los que las empresas privadas parecen darles de modo global más importancia.

Respecto a las fuentes nacionales, se puede decir, que en términos de presencia, las cifras revelan una gran igualdad en el tratamiento al gobierno y a los partidos de la oposición. Destaca la diferencia de la voz de los ciudadanos que se constituyen como fuente en las cadenas públicas casi duplicando el número de veces que aparecen en las privadas.

No obstante, se confirma que los gobiernos obtienen en las cadenas públicas una imagen más positiva que en las privadas, donde los enfoques negativos superan al resto. En las públicas son las noticias neutras las que más abundan de manera global.

En último lugar, se puede afirmar que las pautas del tratamiento de las fuentes para minorías sociales y culturales también adquieren el mismo patrón en públicas y privadas, sin que pueda observarse ninguna diferencia significativa.

\section{ANÁLISIS GLOBAL DE LA MUESTRA}

Considerando las 1346 noticias como una misma muestra, los datos vuelven a confirmar la tendencia a la uniformidad en los comportamientos televisivos. 
Los informativos adquieren carácter nacional en un 70,1\% de los casos, ya que las noticias tienden a retransmitirse desde el propio país. El segundo foco de noticias está en la Unión Europea con un $10,4 \%$ de los casos, y en tercer lugar aparece EEUU con un $7,9 \%$.

En cuanto a los temas, encabezan la lista las cuestiones relacionadas con "crimen" (en esta semana recordemos que hay varios sucesos de estas características) y "deportes" con un 12,6\% y un $12,1 \%$ respectivamente. A continuación son las políticas gubernamentales las que más acaparan la atención informativa $(7,9 \%)$ y los desastres $(7,2 \%)$. Cuestiones militares y partidos políticos adquieren también un protagonismo significativo. Se puede afirmar que los informativos reparten bastante su tiempo entre tres cuestiones básicas: sucesos, deportes y política del gobierno.

Respecto a las fuentes, aparecen muy pocas de carácter internacional, y son de nuevo, las fuentes locales las que tienen presencia y voz en los informativos. En este caso, el protagonismo indudable lo tiene el gobierno del país (7,4\% de las noticias), ya sea presentando una política o defendiéndose de alguna acusación (en la mayoría de los casos). La segunda fuente más consultada en términos globales son los propios ciudadanos del país, y sólo después aparecen los partidos de la oposición. No obstante, hay que señalar, que en cuestión de imagen o enfoque, el gobierno tiende a salir reflejado de manera neutra o negativa en la mayoría de los casos.

Finalmente, en cuanto a las minorías sociales y culturales, es destacable su escasa presencia. Pero, cuando aparecen, la fuente suele ser el periodista, por encima de la propia minoría o de los políticos, que tienen porcentajes similares de aparición en estos temas. En las cuestiones culturales, los expertos tienen también voz, pero de modo muy minoritario.

\section{TENDENCIAS GENERALES DE LOS INFORMATIVOS EUROPEOS}

El análisis de los datos arroja una serie de tendencias relevantes en los informativos de los cinco países examinados que se comentan a continuación.

1. Un marcado localismo incluso en el tratamiento de la información internacional.

En este sentido, se observa, por ejemplo, que es bastante notable la ausencia de un pluralismo en relación con voces exteriores (Estados Unidos y otros estados de la Unión Europea). Se impone, en todos los casos observados, una visión nacional.

Las diferencias nacionales más significativas se producen en relación con dos cuestiones: la presencia de los actores internacionales y el tratamiento concedido a las minorías.

En el primer caso, se reflejan las prioridades e intereses de la política exterior (España, Italia y Francia) con la excepción de Reino Unido que parece considerar esas prioridades cuestiones de estado: el hecho de que en la información internacional recoja noticias de otras áreas diferentes 
de Estados Unidos y la Unión Europea refleja la composición de su sociedad - asiáticos, africanosmás que los intereses políticos.

2. Desde el punto de vista cuantitativo, es destacable la presencia equilibrada de fuentes gubernamentales y de la oposición, tanto en la televisión pública como en la privada.

Las diferencias mas señaladas aparecen en el análisis cualitativo, que demuestra que la imagen de los gobiernos en las televisiones públicas tiene un tono más positivo.

Asimismo, la legislación, que ha venido entendiendo de modo mayoritario el pluralismo de los contenidos como una representación de las fuerzas políticas, parece haber calado en los contenidos de los informativos, puesto que predomina el reparto de las fuerzas políticas, con una importante influencia gubernamental, más destacada en el caso de las públicas.

3. En el tratamiento de las minorías se aprecian diferencias en la presencia o representación, pero un comportamiento homogéneo en lo que respecta a la ausencia de acceso directo.

4. En la televisión privada, se refleja una cierta tendencia al sensacionalismo en la selección de los temas.

\section{NUEVAS DIMENSIONES DEL PLURALISMO}

El estudio demuestra que los informativos europeos, cumpliendo con la normativa establecida para defender el pluralismo, muestran insuficiencias notables en aspectos que son esenciales para garantizar la defensa de este principio.

La primera insuficiencia es que la legislación tiene un concepto excesivamente político del pluralismo. Regula solo la presencia de los diversos actores políticos nacionales y las televisiones cumplen reflejando una representación equilibrada. Sin embargo, desatiende a los actores políticos internacionales y a los actores sociales y culturales cuya representación resulta escasa y desproporcionada.

La segunda insuficiencia es el resultado de evaluar exclusivamente la presencia en los medios sin tener en cuenta los nuevos elementos que introduce el tratamiento mediático. El análisis de la naturaleza de la intervención en los diversos sectores estudiados y el enfoque con que se proyectan pueden producir discriminaciones en la imagen pública de esos protagonistas.

Parece necesario, por tanto, plantear un concepto de pluralismo que considere la presencia de actores distintos de los propiamente políticos y que profundice más en la línea del tratamiento de los contenidos propia de los medios de comunicación. 


\section{REFERENCIAS BIBLIOGRÁFICAS}

- Albarran, A. (2002): Media Economics: Understanding Markets, Industries and Concepts, Ames, lowa State Press.

- Burgelman, Jean Claud \& Pauwels, Caroline, (1992): "Audiovisual policy and cultural identity in small european states: the challenge of a unified market", Media, Culture and Society, vol.14, pp.169-183.

- Consejo de Europa (1994): "4éme Conference Ministerelle Europeenne sur la Politique des communications de masse. Les media dans une société démocratique". Praga, 7-8.XII. 1994. MCM (94)5. Estrasburgo: Consejo de Europa.

- Doyle, Gillian (2002): Media Ownership: concentration, convergence and public policy, Sage.

- La Porte, Ma Teresa y Sádaba, Teresa (2002): "Globalización y diversidad cultural en la política audiovisual europea", Comunicación y Sociedad, pp. 101-127.

- Machet, Emmanuelle, (1998): Television and culture: policies and regulations in Europe, European Institute for the Media.

- Nieto, A.; Iglesias, F. (2000): La Empresa Informativa, Barcelona: Ariel.

- Picard, R. (2002): The Economics and Financing of Media Companies, New York: Fordham University Press.

- Pitkin, Hanna F.(1985): El concepto de representación. Madrid: Centro de Estudios Constitucionales.

- Sánchez-Tabernero, Alfonso; Carvajal, Miguel (2002): Media Concetration in the European Market. Trends and Challenges, Pamplona: Media Market Monograph.

- Shaughnessy, Haydn, (1990): The cultural obligations of broadcasting: national and transnational legislation concerning cultural duties of television broadcaster in Europe, Manchester: European Institute for the Media.

- Toussaint, N. (1996): L'economie de medias, Paris: Press Universitaires de France.

- Unesco (2001): Informe Mundial sobre la cultura 2000/2001. Diversidad Cultural, Conflicto y Pluralismo. Ediciones UNESCO.

- Vallés, Josep M. y Bosch, Agustí (1997): Sistemas electorales y gobierno representativo. Barcelona: Ariel.

- Wright, Paul L. (1994): "Attacking global markets or defending cultural diversity?", Intermedia, Vol. 22 (3), pp. $10-11$. 